\title{
NEW TECHNIQUE FOR BUNCH SHAPE FLATTENING
}

\author{
A. Blas, S. Hancock, M. Lindroos, F. Pedersen, CERN, Geneva, Switzerland, \\ S. Koscielniak, TRIUMF, Vancouver B.C., Canada
}

\begin{abstract}
A technique for increasing the bunching factor $\left(B_{f}\right)$ is described. Typically in booster-type synchrotrons, it is important to reduce the transverse space-charge tune shift. One means to achieve this is to increase the ratio of average to peak longitudinal charge density. Essentially, the idea is to create hollow bunches by sweeping highharmonic empty buckets into the particle beam prior to bunching and acceleration. Successful beam experiments are reported with supporting LONG1D simulation studies performed on the CERN PS Booster for both single and dual rf cases. The longitudinally hollow bunches also benefit the receiving ring during the double batch transfer where half of the PS has to wait 1.2 seconds at low energy for the second injection. A $15^{\text {th }}$ harmonic rf system was used to form the empty buckets. Simulations show that for the single harmonic case, $B_{f}$ is increased from 0.28 to 0.38 , and for the dual harmonic one, $B_{f}$ is increased from 0.45 to 0.55 (values at $100 \mathrm{MeV}$ ). The flattening technique has been tested successfully with the first harmonic to $1 \mathrm{GeV}$ and to $100 \mathrm{MeV}$ for dual harmonic acceleration.
\end{abstract}

\section{INTRODUCTION}

Phase space displacement acceleration of a coasting beam by decelerating empty buckets through it has been described by Dome [1]. Perhaps the best picture is to think of the beam particles sliding up between the empty buckets as these descend, Figure 1. Depositing empty buckets into the core of a coasting beam and then turning the $\mathrm{rf}$ off will give rise to a double peaked energy spectrum, Figure 2. Capture of this beam into a low harmonic bucket and subsequent filamentation will give rise to a hollow phase space distribution, Figure 4. This technique was tested at the Booster in 1979 [2], but was limited by intensity-related problems. Since that time, rf feedback has been implemented; $h=1$ replaced $h=5$; and the bucket area doubled, so the enhanced space charge from a hollow bunch is more tolerable. Further, in 1992 hollow distributions were accelerated in the PS [3] providing the incentive to renew attempts in the Booster.

\subsection{Slew rate}

Let $\Delta \omega$ be the angular frequency sweep, $T$ the duration of the sweep and $\omega_{s}$ the angular synchrotron frequency at the high harmonic. All the standard formulae for rf buckets can be adopted parametrized in terms of the synchronous phase given by $\sin \Phi_{s}=-\Delta \omega /\left[\omega_{s}^{2} T\right]$.
Here $\Delta \omega$ must be sufficient to place the initial buckets outside the beam and so, for given voltage, $T$ is the only free variable with which to control the height and width of the buckets and the adiabaticity of the process. $T$ is limited by the need to accelerate away from space-charge induced transverse betatron resonances. Longitudinal space charge also has an influence: it reduces the size of full buckets and increases the size of empty ones.

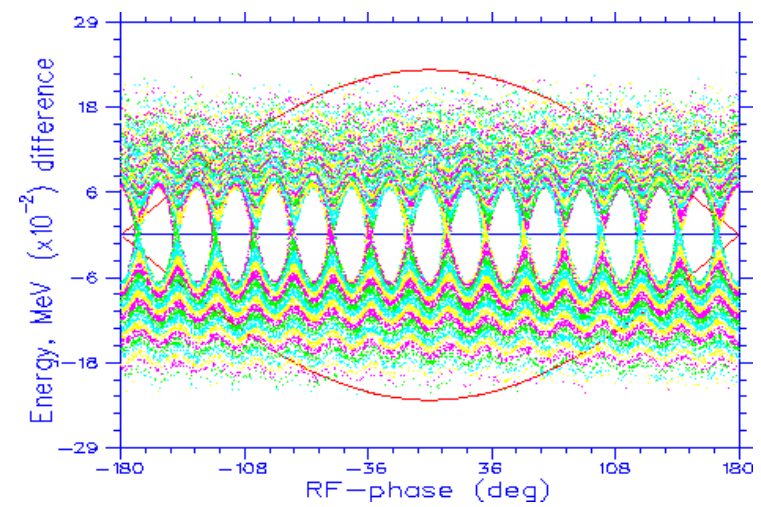

Figure 1: Computer simulation of longitudinal phase space after deposition of $V_{16}=1 \mathrm{kV}$ empty buckets; $T=5 \mathrm{~ms}$.

\subsection{CERN PS Booster}

The PS Booster has three rf systems: C02 $h=1, \mathrm{C} 04 h=2$ or $h=4$, and the C16 system capable of producing harmonics from 10-27 at $50 \mathrm{MeV}$. $\mathrm{C} 02$ and $\mathrm{C} 04$ operate at up to 8 and $5 \mathrm{kV}$, respectively. For C16 at 1 to $4 \mathrm{kV}$, sweeps of $20-30 \mathrm{kHz}$ in 20 to $5 \mathrm{~ms}$ are appropriate, followed by capture in 2 to $6 \mathrm{~ms}$ depending on how short is the $50 \mathrm{MeV}$ injection porch. To achieve a uniform phase depopulation, it is preferable to use a high harmonic number for the buckets; the strong rf feedback on the C16 system makes this an easier proposition.

\section{BTFM}

In principle, the $\mathrm{C} 16 \mathrm{rf}$ manipulations can and have been carried out [4] using dead reckoning. However, in the PS Booster it is possible to use a beam transfer function measurement [5], BTFM, to diagnose the precise energy at which the buckets are deposited via the notch introduced into the energy spectrum of the coasting beam, Figure 2. The C04, $h=4$, cavity is used to tickle the beam with noise. The beam response is proportional to the derivative of the particle density distribution per unit energy. Reconstructing the distribution requires integration of a very noisy response spectrum and leads to a stochastically varying baseline. 


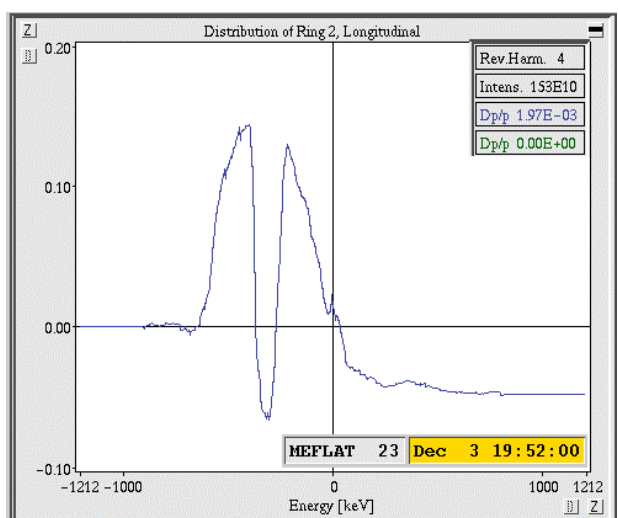

Figure 2: Energy spectrum (from BTFM) after deposition of empty buckets with $V_{15}=4 \mathrm{kV}, 8 \mathrm{~ms}$ frequency sweep.

\subsection{Initial energy spectrum}

Based on the principle that one cannot introduce a notch where there are no particles, one may calibrate the BTFM by sweeping small empty buckets (e.g., $V_{16}=0.5 \mathrm{kV}$ ) of known $\mathrm{rf}$ into the periphery of the beam and looking for the notches. After calibration, the BTFM with C16 off can be used to obtain the beam energy spectrum versus injected intensity. Figure 3 shows three measures of width: $100 \%$ full width, full width without tails and FWHH.

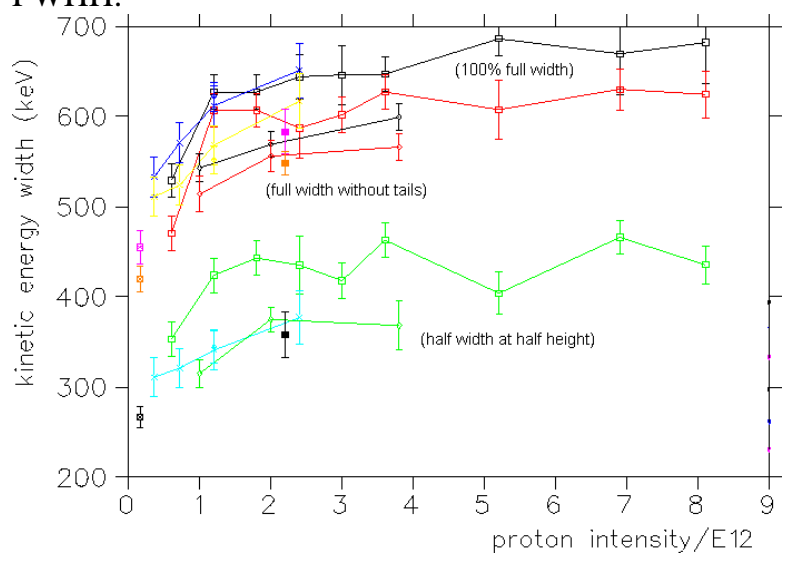

Figure 3: Beam energy width versus injected intensity.

It is clear that the beam is initially much wider than the $370 \mathrm{keV}$ measured by spectrometer, at the exit from the linac, and assumed in the computer simulations.

\section{SINGLE HARMONIC}

The hollow bunch technique has been refined in several machine development periods and complemented with computer simulations [6]. Experimental work has benefited greatly from another diagnostic technique: beam tomography [7] of bunch shape data that spans at least half a synchrotron period. The following data, acquired in experiments spanning several months, shows successful capture and acceleration of the hollow beam to $1 \mathrm{GeV}$. Results are not always reproducible, though the BTFM has helped in this respect. One cause of variability that has been identified is the $180^{\circ}$ phase jump of the fundamental during locking to the beam after the $\mathrm{C} 16$ modulation produces slightly asymmetric double-peaked bunch shapes.

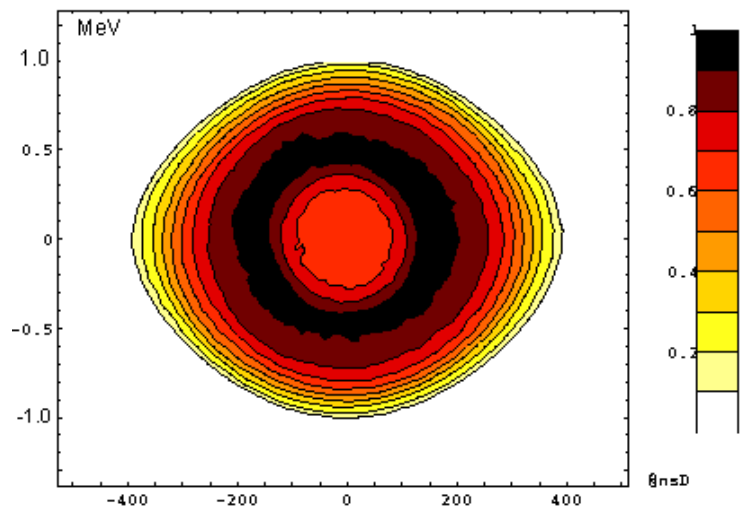

Figure 4: Tomographic reconstruction of phase space after $h=1$ capture and acceleration to $100 \mathrm{MeV}$ flat top; $V_{16}=$ $2 \mathrm{kV}$, frequency sweep in $20 \mathrm{~ms}$, intensity $7 \times 10^{12} \mathrm{ppp}$.

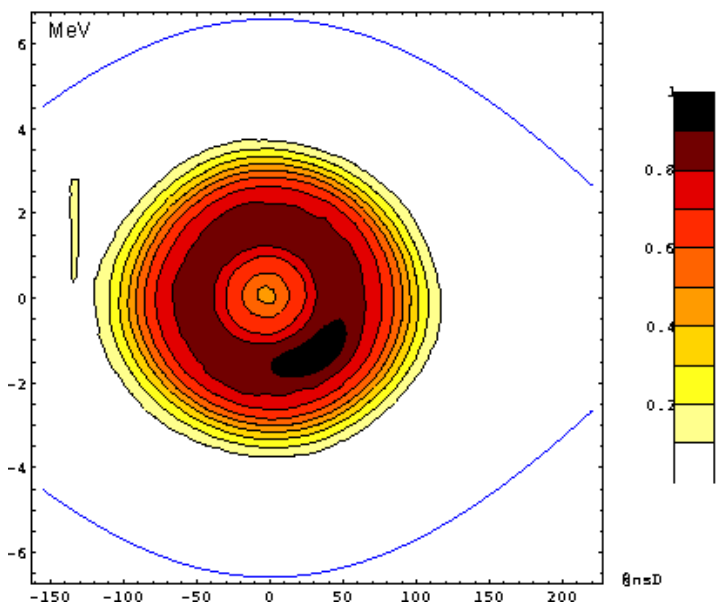

Figure 5: Tomographic reconstruction of phase space after acceleration to $1 \mathrm{GeV}, V_{15}=3 \mathrm{kV}, 8 \mathrm{~ms}$ sweep, $6 \times 10^{12} \mathrm{ppp}$.

Because of the increased emittance and the typically flat or double-peaked bunch shape, the peak charge densities are reduced and the bunching factor increased.

Table 1: Bunching factors at $100 \mathrm{MeV}$, single harmonic.

\begin{tabular}{|c|c|c|}
\hline C16 & $B_{f}$ prediction & $B_{f}$ measurement \\
\hline Off & 0.29 & $0.32-0.34$ \\
\hline On=1kV & 0.36 & 0.37 \\
\hline On $=2 \mathrm{kV}$ & 0.39 & $0.37-0.39$ \\
\hline On=4kV & $?$ & $0.39-0.41$ \\
\hline
\end{tabular}

\section{DUAL HARMONIC}

Setting up for dual harmonic operation is complicated by the fact that the $\mathrm{C} 04$ system is to be used both for the BTFM ( $h=4)$ and the second harmonic $(h=2)$, though not in the same magnetic cycle. Adjustment of the empty bucket deposition is more delicate and, typically, a dipole 
component remains after capture by the dual harmonic rf, Figure 6. Acceleration to $100 \mathrm{MeV}$ is possible at low intensity, Figure 8, but a longitudinal instability hinders further acceleration due to losses. Up to this energy, experimental data compares quite favourably with simulation [6], Figure 8, Figure 9 and Table 2.

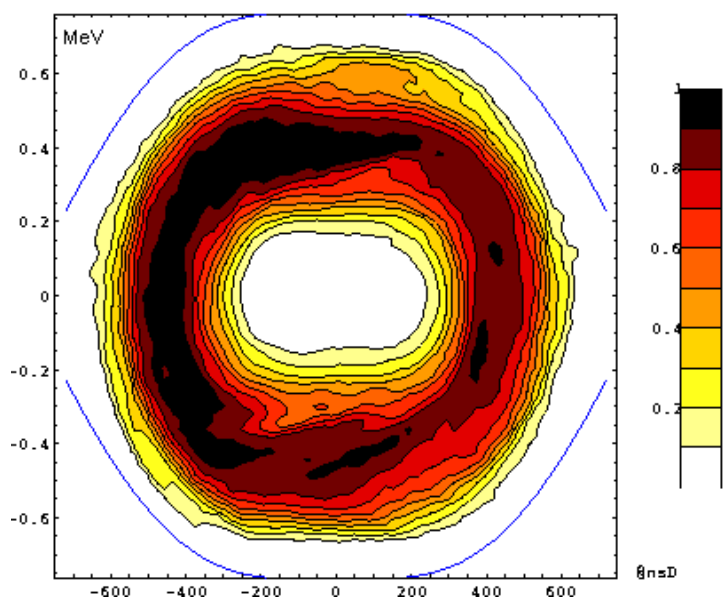

Figure 6: Tomographic reconstruction at $50 \mathrm{MeV}, 20 \mathrm{~ms}$ filamentation $V_{15}=4 \mathrm{kV}, 8 \mathrm{~ms}$ sweep, intensity $2 \times 10^{12} \mathrm{ppp}$.

Table 2: Bunching factors at $100 \mathrm{MeV}$, dual harmonic.

\begin{tabular}{|c|c|c|}
\hline $\mathrm{C} 16$ & $\mathrm{~B}_{\mathrm{f}}$ prediction & $\mathrm{B}_{\mathrm{f}}$ measurement \\
\hline Off & 0.44 & $0.44-0.46$ \\
\hline $\mathrm{On}=2 \mathrm{kV}$ & 0.51 & $0.46-0.48$ \\
\hline $\mathrm{On}=4 \mathrm{kV}$ & 0.55 & $0.46-0.52$ \\
\hline $\mathrm{On}=6 \mathrm{kV}$ & 0.59 & $0.53-0.57$ \\
\hline
\end{tabular}

\subsection{Longitudinal instability}

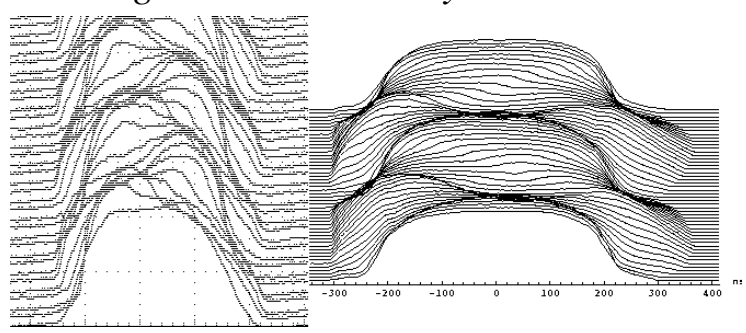

Figure 7: Sextupole mode at $120 \mathrm{~ms}$, octupole at $160 \mathrm{~ms}$.

Careful observations show the instability to be present even when $\mathrm{C} 16$ is turned off and the bunches are not hollow, but in this case the instability is less severe and usually the losses are not significant. The upward ramp of the Booster magnet cycle lasts $\approx 500 \mathrm{~ms}$. The instability starts at $\approx 50 \mathrm{~ms}$ and stops at $\approx 250 \mathrm{~ms}$. During this time, at least two within-bunch oscillation modes appear to be present: first sextupole and then octupole, Figure 7. It is suspected that the hollow amplitude distribution contributes unfavourably to the growth rate. When the instability is over, the distribution of the surviving particles is monotonically decreasing with amplitude. The instability appears related to the control loop which synchronises second harmonic to the fundamental waveform. Opening this loop calms the instability, but loss of $h=1,2$ relative phasing eventually leads to beam loss.

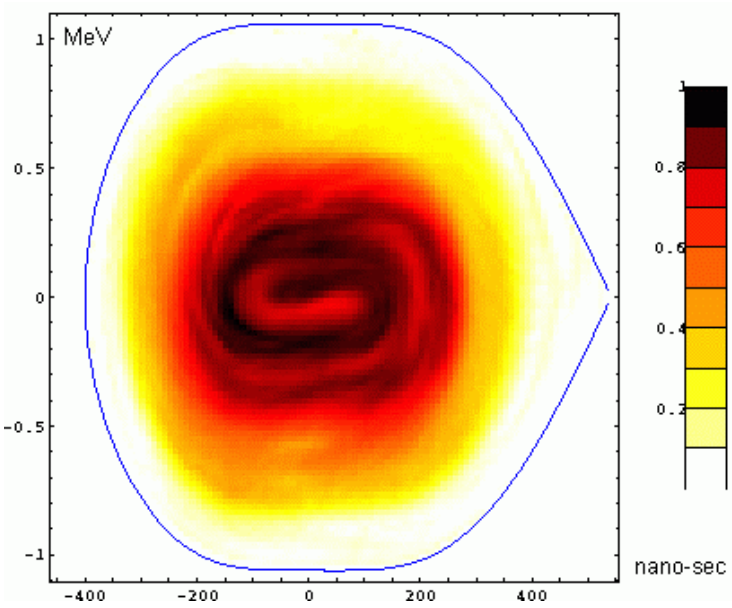

Figure 8: Tomographic reconstruction of phase space at $120 \mathrm{MeV}, V_{15}=4 \mathrm{kV}, 6 \mathrm{~ms}$ frequency sweep, $10^{12} \mathrm{ppp}$.

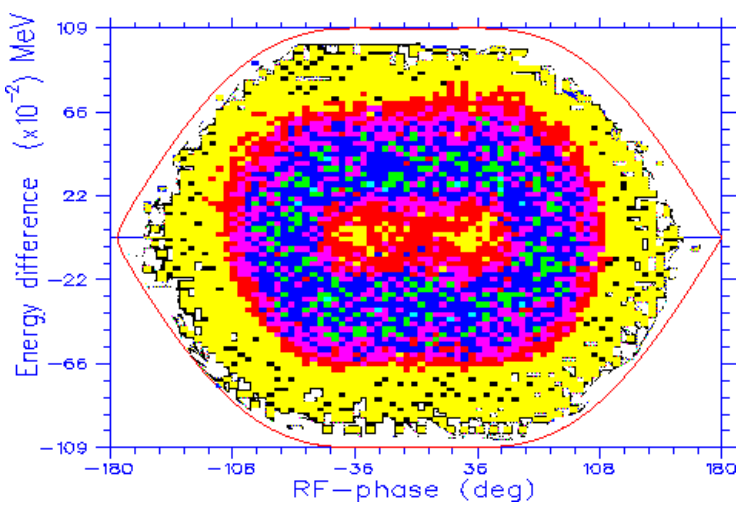

Figure 9: Computer simulation, $105 \mathrm{MeV}$ flat top, $V_{16}=2$ $\mathrm{kV}, 8 \mathrm{~ms}$ frequency sweep, intensity $3 \times 10^{12} \mathrm{ppp}$.

\section{CONCLUSION}

With relatively little effort, this technique of bunch flattening has been successfully applied to single harmonic acceleration. Progress with dual-harmonic hollow bunches has been compromised by an instability. However, the evidence suggests that this problem is particular to the PS Booster low-level rf and may not be a hindrance at other machines.

\section{REFERENCES}

[1] G. Dome: Theory of rf acceleration, CERN 87-03.

[2] J. Delahaye et al: Shaping of proton distribution, Proc $11^{\text {th }}$ Int. Conf. on High-Energy Accels, Geneva, pp.299-304, 1980.

[3] R. Cappi et al: Proc. 1993 PAC, Washington D.C., p.3570.

[4] A. Blas: CERN/PS/RF/Note 98-14 (MD).

[5] H. Schonauer: PS/OP/Note 96-18 \& PS/OP/Note 97-52.

[6] S. Koscielniak: TRI-DN-99-13 \& TRI-DN-99-14.

[7] S.Hancock, M.Lindroos, E.McIntosh, M.Metcalf: Tomo-graphic measurements of longitudinal phase space density, accepted for publication in Computer Physics Communications. 